\title{
Steroid Induced Psychosis in a Rare Case of Bronchiolitis Obliter- ans Organizing Pneumonia
}

\author{
Bryn Dhir ${ }^{*}$ and Rachit Kumar ${ }^{2}$ \\ ${ }^{1}$ International American University College of Medicine, USA \\ ${ }^{2}$ Georgia Institute of Technology, USA
}

*Corresponding author: Bryn Dhir, MS IV, Year IV Medical Student, c/o International American University College of Medicine, 1255 W 15th Street, Suite 100, Plano, Texas 75075, USA, Tel: 312-918-2454, Fax: 972-408-3457

\begin{abstract}
We report a case of an 84-year-old female with altered mental status and known Bronchiolitis Obliterans Organizing Pneumonia suspected from high dose steroid who presented for medical management of acute psychosis. This case centers on the inpatient management of older patients in the setting of steroid use with consideration given to dosing, supportive care and therapy during management.
\end{abstract}

\section{Keywords}

Bronchiolitis obliterans organizing pneumonia, Chronic obstructive pulmonary disease, Corticosteroids, Psychosis, Pulmonary, Psychiatry

\section{List of Abbreviations}

BOOP: Bronchiolitis Obliterans Organizing Pneumonia; COP: Cryptogenic Organizing Pneumonia; COPD: Chronic Obstructive Pulmonary Disease; ED: Emergency Department; SLE: Systemic Lupus Erythematosus

\section{Introduction}

latrogenic psychiatric conditions due to therapeutic medical management of pulmonary diseases can be complicated when options for medical management is limited. This may be further complicated with increased patient age, comorbidities, and other underlying psychiatric conditions such as depression. Medications causing psychiatric conditions, delusions, hallucinations, psychosis, memory loss and confusion have been reported with the use of steroids, and acute psychosis secondary to high dose steroid use is among the known complications in the management of patient diseases. To the best of our knowledge, we report the first to case to dis- cuss the management of steroid induced psychosis in a patient with the presentation of a rare pulmonary disorder, bronchiolitis obliterans organizing pneumonia (BOOP), and we highlight the importance appropriately managing patients with this disease which is otherwise limited in treatment options.

\section{Case Description}

An 84-year-old female with a past psychiatric history of major depression and anxiety presented with new onset psychotic features, depression and fixed paranoid delusions and agitation, was brought into the Emergency Department (ED) from a long term care facility. The patient indicated that the staff at the facility was attempting to poison her food, and that another resident had been heating up her chair which was causing electric shocks up her legs and around her groin. Triage and repeat vitals: temperature $98.5 \mathrm{~F}$; respiratory rate: $20 \mathrm{bpm}$; pulse rate: 90 bpm; blood pressure: 138/70 $\mathrm{mmHg}$; were within normal limits. Prior to acute onset, the patient was described as pleasant and had been enjoying the facility and this was the first occurrence of psychiatric changes. The episode was further associated with multiple attempts of eloping from the facility. The patient reported poor sleep, decreased energy, depressed mood, lack of appetite and psychomotor decline. She denied visual or auditory hallucinations or suicidal ideation.

Medically of note, the patient had a history of chronic obstructive pulmonary disease (COPD), asthma, depression, anxiety disorder, dysphagia, poly-

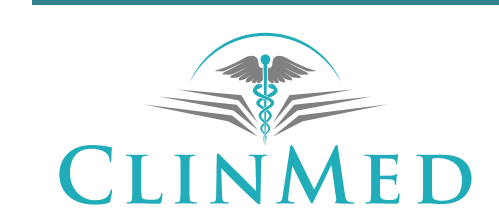

INTERNATIONAL LIBRARY 
neuropathy, a history of smoking, hyperglycemia, hypothyroid, and a nine year history of bronchiolitis obliterans organizing pneumonia (BOOP) for which she was hospitalized eight months prior for complications. During that time, she was bedridden for three weeks and was prescribed $250 \mathrm{mg}$ of Azithromycin every other day for two weeks, and $40 \mathrm{mg}$ of Prednisone once a day. During that time her depression and mental state progressively deteriorated. Approximately one week prior to presentation in the ED, a management plan was initiated to taper down the $40 \mathrm{mg}$ of Prednisone, to $20 \mathrm{mg}$ once a day for thirty days, and was to be followed by a further decrease to $10 \mathrm{mg}$ once a day for thirty days while continuing to monitor for lung infection. The patient continued her medications for chronic medical history. Of note, the medications included: $0.63 \mathrm{mg} / 3 \mathrm{~mL}$ albuterol nebulizer solution every 4 hours as needed for wheezing; $10 \mathrm{mg}$ Montelukast nightly for asthma; $0.25 \mathrm{mg} / 2 \mathrm{~mL}$ budesonide nebulizer suspension two times a day for bronchitis; $0.5 \mathrm{mg}$ Alprazolam taken by mouth nightly for anxiety; $7.5 \mathrm{mg}$ Buspirone twice a day for anxiety; $126 \mathrm{mcg}$ Levothyroxine once a day for hypothyroidism; Lantus insulin for management of hyperglycemia; 10-325 mg Oxycodone-Acetaminophen tablet taken by mouth every six hours for pain; $100 \mathrm{mg} \mathrm{Gab}$ apentin capsule taken at lunch time and a $100 \mathrm{mg} \mathrm{Ga-}$ bapentin capsule taken at nighttime for neurogenic pain; and Continuous Positive Airway Pressure (CPAP) therapy for obstructive sleep apnea.

Medical imaging of a chest X-ray showed: Opacification of bilateral hemidiaphragms with mild patchy bibasal densities compatible with pneumonia; small bilateral blunting of the costophrenic angles and pleural effusions compatible with pneumonia and atelectasis; mild cardiomegaly; mild degree of osteoporosis; and mild osteoarthritis. Investigation of hematochemical parameters indicated: white blood cell count: 13.1; Hemoglobin: 11.6; Hematocrit: 37.0; Platelet Count: 237,000; Sodium: 140; Potassium: 2.9; Chloride: 99; Carbon Dioxide: 34; Blood Urea Nitrogen: 14; Creatine: 0.9; Glucose: 54; Calcium 9.2. Further evaluation with the brief interview for Mental Status showed a total score of 15/15, indicating the patient was cognitively intact.

The patient was hospitalized in a psychiatric facility for management. She made good eye contact, maintained an appetite, sleep, and was cooperative. After twenty-two days of hospitalization, the patient's paranoia decreased, and the psychosis had resolved. She was discharged to her long term care facility with treatment follow up instructions, management plan, and education.

\section{Discussion}

Bronchiolitis obliterans organizing pneumonia (BOOP), also referred to as Epler's pneumonia, is a rare lung disorder with the distinguishing trait of simultaneous inflammation of the bronchioles and alveolar sacs [1]. General pneumonias are distinguished by granulation tissue in the distal air space, however in the case of associated granulation tissue within the bronchiolar lumen, the term bronchiolitis obliterans [2] allowing for it to be uniquely distinguished histologically and radiographically [3] from idiopathic pulmonary fibrosis. In cases where the etiology of BOOP is known, it is classified as secondary $\mathrm{BOOP}$ or as cryptogenic organizing pneumonia (COP), cryptogenic organizing pneumonitis, or idiopathic BOOP if unknown [4-6]. The cause of BOOP is generally unknown however, secondary BOOP has been attributed to viral infection, iatrogenesis from other medical management, or underlying conditions such as irritable bowel or connective tissue disorders $[7,8]$. Distressing complaints of BOOP commonly include cough, fever, and dyspnea as well as chest pain and hemoptysis and indicates prompt symptomatic relief. The diagnosis of BOOP can be difficult due to the varying profiles of disease presentation as well as the presence of other overlying interstitial lung disorders, therefore a thoracoscopic lung biopsy is required for direct visualization [9-11].

The established treatment of BOOP is corticosteroids, in particular prednisone, however dosage schedules have yet to be standardized. Typically, physicians have reported tapered doses of prednisone over the course of several months, and in some cases, treatment has been for over a year $[12,13]$. Relapse is a possibility in $30 \%$ of patients and has been associated with additional complications including pneumothorax and lead to a longer duration of treatment $[14,15]$ Despite relapse potential, most patients respond well to corticosteroid treatment and recover $[3,14,16]$.

Corticosteroids are commonly used in the management of pulmonary and autoimmune conditions and the use is carefully considered for patient age, comorbidities, immunocompetency and disease pathology as a side effect of prolonged use can lead to unfavorable results effecting bone growth, changes to hormone levels, further complicated immunosuppression and a wide range of psychiatric side effects, including disturbance of mood cognition, sleep, and behavior, euphoria, hypomania, frank delirium and psychosis. Of these, steroid induced psychosis is an iatrogenic condition predominantly affecting adults and at lower rates in children [17-20]. The initial recommended intervention for patients presenting with corticosteroid-induced psychosis is cessation of the corticosteroid. In patient cases in which acute steroid cessation is not possible, as was seen in our patient, dose tapering is recommended $[21,22]$.

Palliative pharmacotherapy with mood stabilizers and antipsychotics was also indicated in management therapy of our patient's acute episode of psychosis and 
intolerable distress due to other comorbidities [19].

In a similar case previously reported, a 46-year-old woman with systemic lupus erythematosus (SLE) being treated with prednisolone $40 \mathrm{mg}$ daily for exacerbated erythema, was diagnosed with suffering from steroid-induced psychosis and was unable to cease prednisolone due to the exacerbation. Prednisolone dosage was tapered, and the patient was treated with valproic acid $800 \mathrm{mg}$ and risperidone $4 \mathrm{mg}$ daily until cessation of symptoms a week later when the dosage of prednisolone had been tapered to $22.5 \mathrm{mg}$. In this case, the choice of therapy with valproic acid and risperidone was based on the patient's diagnosis of SLE, which could lead to further complications with other therapies [23]. Further, the patient in this case report contrasts to previous reports indicating the resolution of psychosis achieved when the steroid has been completely stopped $[19,23]$. Nonetheless it is important to note, for patients receiving high dose or long-term treatment of corticosteroids, corticosteroid withdrawal is a necessary consideration when managing the duration for dose tapering.

\section{Conclusion}

BOOP is a rare pulmonary disease with complicated pathology and with no standardized therapy and infections and acute exacerbations are known complications and patients require closely monitored, patient specific medical management. For medical conditions such as this, treatment and management options are limited, and steroid therapy is required for management and for the prevention of further complications. However, the use of corticosteroids may induce psychosis, and management relies on multifactorial contributors including the underlying condition in which it is originally prescribed. This case report highlights the importance of increased age, comorbidities, duration and dosing increments of managing the rare medical condition of bronchiolitis obliterans organizing pneumonia.

\section{Funding}

There is no funding to report.

\section{Acknowledgements}

BD would like to thank Dr. Edward Swanton, psychiatrist in Baltimore Maryland, for his expertise, support and guidance in patient care.

\section{Ethics Approval and Consent to Participate}

Not applicable.

\section{Consent for Publication}

The patient consents to publish her data.

\section{Competing Interests}

The authors declare that they have no competing in- terests.

\section{Authors' Contributions}

BD prepared the final manuscript. All authors contributed to the case report and read and approved the final manuscript.

\section{References}

1. Epler GR (2001) Bronchiolitis obliterans organizing pneumonia. Arch Intern Med 161: 158-164.

2. Epler GR, Colby TV, McLoud TC, Carrington CB, Gaensler EA (1985) Bronchiolitis obliterans organizing Pneumonia. N Engl J Med 312: 152-158.

3. Colby TV (1998) Bronchiolitis: Pathologic considerations. American Journal of Clinical Pathology 109: 101-109.

4. Drakopanagiotakis F, Paschalaki K, Abu-Hijleh M, Aswad B, Karagianidis N, et al. (2011) Cryptogenic and secondary organizing pneumonia: Clinical presentation, radiographic findings, treatment response, and prognosis. Chest 139: 893-900.

5. Davison AG, Heard BE, McAllister WA, Turner-Warwick ME (1983) Cryptogenic organizing pneumonitis. Q J Med 52: 382-394

6. Cordier JF, Loire R, Brune J (1989) Idiopathic bronchiolitis obliterans organizing pneumonia. Definition of characteristic clinical profiles in a series of 16 patients. Chest 96: 999-1004.

7. Epler GR (2013) Post-breast cancer radiotherapy bronchiolitis obliterans organizing pneumonia. Expert Rev Respir Med 7: 109-112.

8. Vasu TS, Cavallazzi R, Hirani A, Sharma D, Weibel SB, et al. (2009) Clinical and radiologic distinctions between secondary bronchiolitis obliterans organizing pneumonia and cryptogenic organizing pneumonia. Respir Care 54: 10281032.

9. Oymak FS, Demirbas HM, Mavili E, Akgun H, Gulmez I, et al. (2005) Bronchiolitis obliterans organizing pneumonia. Clinical and roentgenological features in 26 cases. Respiration 72: 254-262.

10. Yebra M, Romero Y, Varela A, Berrocal E (1994) Percutaneous lung biopsy in the diagnosis of bronchiolitis obliterans organizing pneumonia. Chest 105: 972-973.

11. Poletti V, Cazzato S, Minicuci N, Zompatori M, Burzi M, et al. (1996) The diagnostic value of bronchoalveolar lavage and transbronchial lung biopsy in cryptogenic organizing pneumonia. Eur Respir J 9: 2513-2516.

12. Nizami IY, Kissner DG, Visscher DW, Dubaybo BA (1995) Idiopathic bronchiolitis obliterans with organizing pneumonia. An acute and life-threatening syndrome. Chest 108: 271-277.

13. Kofteridis DP, Bouros DE, Vamvakas LN, Stefanaki KS, Voludaki AE, et al. (1999) Pneumothorax complicating fatal bronchiolitis obliterans organizing pneumonia. Respiration 66: 266-268.

14. Cohen AJ, King TE, Jr., Downey GP (1994) Rapidly progressive bronchiolitis obliterans with organizing pneumonia. Am J Respir Crit Care Med 149: 1670-1675.

15. Sara AG, Hamdan AJ, Hanaa B, Nawaz KA (2008) Bronchiolitis obliterans organizing pneumonia: Pathogenesis, clinical features, imaging and therapy review. Ann Thorac Med 3: 67-75. 
16. Epler GR (2011) Bronchiolitis obliterans organizing pneumonia, 25 years: A variety of causes, but what are the treatment options? Expert Rev Respir Med 5: 353-361.

17. Barnes DM (1986) Steroids may influence changes in mood. Science 232: 1344-1345.

18. Warrington TP, Bostwick JM (2006) Psychiatric adverse effects of corticosteroids. Mayo Clinic Proceedings 81: 13611367.

19. Hodgins GE, Saltz SB, Gibbs EP, Gonzalez R, Regan J, et al. (2018) Steroid-Induced Psychosis in the Pediatric Population: A New Case and Review of the Literature. J Child Adolesc Psychopharmacol 28: 354-359.
20. Ahmad M, Rasul FM (1999) Steroid-induced psychosis treated with haloperidol in a patient with active chronic obstructive pulmonary disease. Am J Emerg Med 17: 735.

21. Richter B, Neises G, Clar C (2002) Glucocorticoid withdrawal schemes in chronic medical disorders. A systematic review. Endocrinol Metab Clin North Am 31: 751-778.

22. Kato O, Misawa $\mathrm{H}$ (2005) Steroid-induced psychosis treated with valproic acid and risperidone in a patient with systemic lupus erythematosus. Prim Care Companion J Clin Psychiatry 7: 312.

23. Fricchione G, Ayyala M, Holmes VF (1989) Steroid withdrawal psychiatric syndromes. Annals of Clinical Psychiatry 1: $99-108$. 